\title{
Branching fractions and charge asymmetries in charmless hadronic $B$ decays at BaBar
}

\author{
P. Biassoni on behalf of the BaBar Collaboration \\ Università and INFN, Sezione di Milano - Via Celoria 16, I-20133 Milano, Italy
}

(ricevuto l' 8 Ottobre 2010; pubblicato online l'8 Febbraio 2011)

\begin{abstract}
Summary. - We present measurements of branching fraction and charge asymmetry in charmless hadronic $B$ decays with $\eta$ and $\eta^{\prime}$ mesons in the final state. All results use the final BaBar dataset corresponding to $465 \times 10^{6} B \bar{B}$ pairs.

PACS 13.25. Hw - Decays of bottom mesons.

PACS 12.15. Hh - Determination of Kobayashi-Maskawa matrix elements.

PACS 11.30.Er - Charge conjugation, parity, time reversal, and other discrete symmetries.
\end{abstract}

\section{1. - Introduction}

Experimental measurements of branching fraction $(\mathrm{BF})$ and $C P$-violating charge asymmetry in rare $B$ decays are an important test of the Standard Model (SM) and its extensions. Several predictions are available for these quantities [1,2]. The large BF difference between $\eta^{\prime} K$ and $\eta K$ seems to be explained in the SM context [3]. Rates of the decay modes to $\eta \eta, \eta \phi, \eta^{\prime} \eta^{\prime}$, and $\eta^{\prime} \phi$ are used in flavor $S U(3)$-based calculations [2,4], to constrain the unsigned difference between the $C P$-violating parameter $S$ measured in $\eta^{\prime} K^{0}$ and $\phi K^{0}$ and $\sin 2 \beta$ measured in $J / \psi K^{0}$. The charge asymmetry $\mathcal{A}_{\mathrm{ch}}$ is expected to be sizable in $\eta K^{+}$and suppressed in $\eta^{\prime} K^{+}$decays [2].

\section{2. - Analysis technique}

Results shown in this paper are based on a sample of $465 \times 10^{6} B \bar{B}$ pairs collected at a center-of-mass energy $\sqrt{s}=10.58 \mathrm{GeV}$ at the PEP-II asymmetric $e^{+} e^{-}$collider, and recorded by the $B A B A R$ detector [5]. $B$ meson is reconstructed into several final states with $\eta$ or $\eta^{\prime}$ mesons. These states are listed in table I. $B$ meson is kinematically characterized by $\Delta E \equiv E_{B}-\frac{1}{2} \sqrt{s}$ and $m_{\mathrm{ES}} \equiv \sqrt{s / 4-\vec{p}_{B}^{2}}$, where $\left(E_{B}, \vec{p}_{B}\right)$ is the $B$ meson four-momentum vector expressed in $\Upsilon(4 S)$ rest frame. Background arises primarily from random combinations of particles in $e^{+} e^{-} \rightarrow q \bar{q}$ events $(q=u, d, s, c)$. We suppress this background with requirements on event shape variables and on the energy, invariant mass and particle identification signature of the decay products. For each mode, results are obtained from extended maximum likelihood fits with input variables $\Delta E, m_{\mathrm{ES}}$, and the output of a Fisher discriminant that combines different event shapes variables. Where useful, the masses of $B$ daughters are included in the fit. 
TABLE I. - Results for modes presented in this paper.

\begin{tabular}{l|c|c|c|c}
\hline Decay & $\mathcal{B}\left(10^{-6}\right)$ & $\mathcal{B}$ UL $\left(10^{-6}\right)$ & $S(\sigma)$ & $A_{\text {ch }}$ \\
\hline$\eta \pi^{+}$ & $4.00 \pm 0.40 \pm 0.24$ & - & - & $-0.03 \pm 0.09 \pm 0.03$ \\
$\eta K^{0}$ & $1.15_{-0.38}^{+0.43} \pm 0.09$ & 1.8 & 3.5 & - \\
$\eta K^{+}$ & $2.94_{-0.34}^{+0.39} \pm 0.21$ & - & - & $-0.36 \pm 0.11 \pm 0.03$ \\
$\eta \eta$ & $0.5 \pm 0.3 \pm 0.1$ & 1.0 & 1.9 & - \\
$\eta \omega$ & $0.94_{-0.30}^{+0.35} \pm 0.09$ & 1.4 & 3.7 & - \\
$\eta \phi$ & $0.2 \pm 0.2 \pm 0.1$ & 0.5 & 1.4 & - \\
$\eta^{\prime} \pi^{+}$ & $3.5 \pm 0.6 \pm 0.2$ & - & - & $+0.03 \pm 0.17 \pm 0.02$ \\
$\eta^{\prime} K^{0}$ & $68.5 \pm 2.2 \pm 3.1$ & - & - & - \\
$\eta^{\prime} K^{+}$ & $71.5 \pm 1.3 \pm 3.2$ & - & - & - \\
$\eta^{\prime} \eta^{\prime}$ & $0.6_{-0.4}^{+0.5} \pm 0.4$ & 1.7 & 1.0 & - \\
$\eta^{\prime} \omega$ & $1.01_{-0.38}^{+0.46} \pm 0.09$ & 1.8 & 3.6 & - \\
$\eta^{\prime} \phi$ & $0.2 \pm 0.2 \pm 0.3$ & 1.1 & 0.5 & $-0.08_{-0.018}^{+0.017} \pm 0.009$ \\
\hline
\end{tabular}

\section{3. - Results}

In table I we report the results of $\mathrm{BF}(\mathcal{B})$ and charge asymmetry $\left(\mathcal{A}_{\mathrm{ch}}\right)$ for each decay mode [6]. The first error is statistical and second systematic. Significance $(\sigma)$ is taken as $\sqrt{-2 \ln \mathcal{L}_{\max } / \mathcal{L}_{0}}$, where $\mathcal{L}_{\max }\left(\mathcal{L}_{0}\right)$ is the value of the likelihood at its maximum (for zero signal). Bayesian upper limit at $90 \%$ CL is calculated for modes with significance less than $5 \sigma$. For the well established decay modes $\eta^{(\prime)} K^{+}, \eta^{\prime} K^{0}$, and $\eta^{(\prime)} \pi^{+}$significance is not reported. Main contributions to systematic uncertainties come from fit bias and uncertainties in the probability density functions parameterization. Daughter branching ratios uncertainty limits $\mathrm{BF}$ measurement in $B \rightarrow \eta^{\prime} K$ decay modes.

\section{4. - Conclusions}

We reported measurements for several charmless hadronic $B$ decays. In $B \rightarrow \eta K^{+}$ we find evidence of direct $C P$ violation at $3.3 \sigma$ level.

\section{REFERENCES}

[1] Chen Y. H. et al., Phys. Rev. D, 60 (1999) 094014; Bauer C. W. and Steward I. W., Phys. Lett. B, 516 (2001) 134; XiaO Z. et al., Phys. Rev. D, 75 (2007) 014018.

[2] Beneke M. and Neubert M., Nucl. Phys. B, 675 (2003) 333; Chiang C. W. et al., Phys. Rev. D, 70 (2004) 034020 [and references therein].

[3] Lipkin H. J., Phys. Lett. B, 254 (1991) 247; Beneke M. and Neubert M., Nucl. Phys. $B, 651$ (2003) 225.

[4] Grossman Y. et al., Phys. Rev. D, 68 (2003) 015004; Gronau M. et al., Phys. Lett. B, 596 (2004) 107.

[5] Aubert B. et al. (BABAR Collaboration), Nucl. Instrum. Methods Phys. Res. A, 479 (2002) 1.

[6] Aubert B. et al. (BABAR Collaboration), Phys. Rev. D, 80 (2009) 112002. 